\title{
Un « enfer » à la BNU
}

\section{Christophe Didier, Claude Lorentz et Daniel Bornemann}

\section{(2) OpenEdition}

\section{Journals}

Édition électronique

URL : http://journals.openedition.org/rbnu/313

DOI : 10.4000/rbnu.313

ISSN : 2679-6104

\section{Éditeur}

Bibliothèque nationale et universitaire de Strasbourg

\section{Édition imprimée}

Date de publication : 23 mai 2018

Pagination : 92-99

ISSN : 2109-2761

\section{Référence électronique}

Christophe Didier, Claude Lorentz et Daniel Bornemann, « Un « enfer » à la BNU », La Revue de la BNU [En ligne], 17 | 2018, mis en ligne le 05 septembre 2018, consulté le 11 décembre 2020. URL : http:// journals.openedition.org/rbnu/313; DOI : https://doi.org/10.4000/rbnu.313

\section{(c) (i) (3)(2)}

La Revue de la BNU est mise à disposition selon les termes de la Licence Creative Commons Attribution - Pas d'Utilisation Commerciale - Partage dans les Mêmes Conditions 4.0 International. 
PORTFOLIO

\section{Un «enfer » à la $\mathrm{BNU}$}

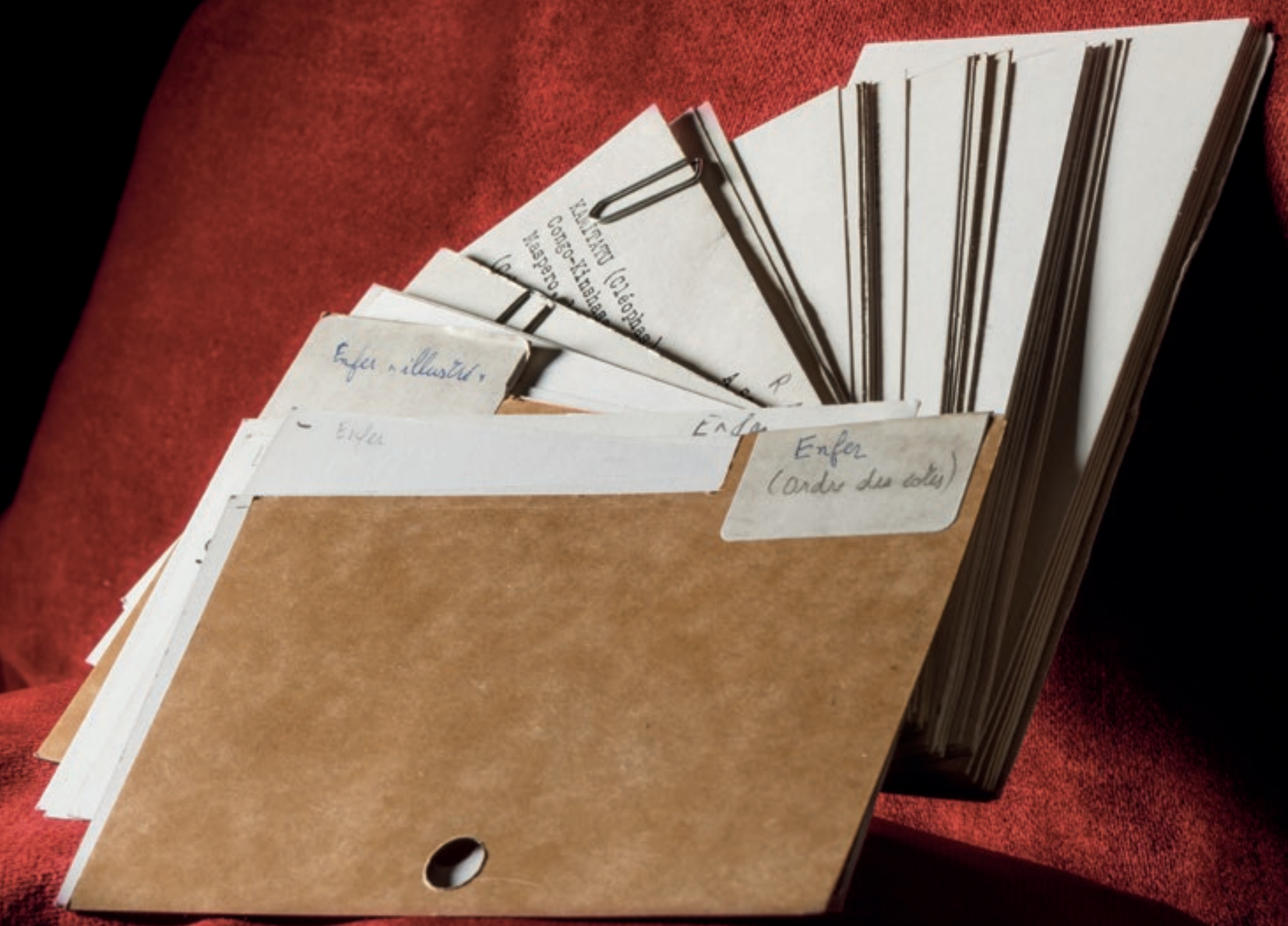


On connaît bien, quand on s'intéresse à l'histoire du livre, la notion d'" enfer " en bibliothèque, cette volonté de classer, ranger, et communiquer à part les ouvrages, illustrés ou non, que la morale (ce qu'on appelait jadis les «bonnes mours») réprouve - ouvrages libertins, galants, légers, érotiques, pornographiques, suivant les termes et les époques qui les emploient. En France, un « enfer » mythique est celui de la BnF, l'ancienne Bibliothèque nationale, qu'une exposition récente ${ }^{1}$ a contribué à remettre sous les feux de la rampe. Institué au milieu du $19^{\text {e }}$ siècle, il est progressivement démantelé après 1968, même si une section spéciale est reconstituée dans les années 80 , mais pour des raisons pratiques de classement et sans difficulté particulière de communication.

À l'heure des commémorations des événements de mai 68 , il est piquant de se ressouvenir que la BNU (ce qui est sans doute moins connu) a constitué son propre " enfer ", mais, semble-t-il, surtout dans ces années soixante et même au cours de la décennie suivante. En attestent aujourd'hui, à côté de la présence dans la Réserve des livres rares et précieux de titres qui étonnent en ce lieu (comme l'édition, très standard, du $\mathrm{Nu}$ dans la peinture paru chez Braun en 1952 et reproduit p. 98), l'existence d'un petit fichier qui témoigne d'une " mise en enfer " instituée, concernant non seulement des titres arrivés par achat ou par le biais du dépôt légal et mis directement en réserve, mais aussi des ouvrages plus anciens (de Crébillon, Vivant Denon, La Sablière ou Apollinaire, entre autres) qui furent décotés pour ne plus être rangés en magasins et donc accessibles sans conditions particulières : une fois recotés en réserve, ils ne devenaient consultables que dans une salle particulière et sous l'œil d'un gardien...

$\mathrm{Au}$ vu de nos connaissances actuelles, nous ne pouvons affirmer que cet " enfer " ait fait l'objet de dispositions spécifiques, consignées dans une note de service ou au sein d'un règlement. On ne sait pas non plus si, à l'instar de ce qui se faisait à la $\mathrm{BnF}$ au $19^{\mathrm{e}}$ siècle, la consultation des ouvrages incriminés passait par une autorisation d'un bibliothécaire. Un travail de recherche devra être mené dans les archives de la BNU. Le fichier lui-même est par contre déjà très parlant. Il est divisé en deux parties inégales : celle intitulée " Enfer (ordre des cotes) » semble contenir le petit groupe de titres, mentionnés plus haut, qui ont été décotés dans les années 70 ; la mention «Enfer " est systématiquement portée sur les fiches (voir ill. p. 95 ou 96, où l'on aperçoit clairement l'ancienne cote, gommée). La partie, beaucoup plus importante, intitulée « Enfer 'illustré' », avec une fiche précisant les modalités de constitution de ce fonds (reproduite p. 94 en bas) ${ }^{2}$, comprend plusieurs types d'ouvrages : quelques titres traitant de politique africaine et émanant d'opposants aux régimes en place, avec lesquels la France entretenait des relations diplomatiques, ce qui justifiait, aux yeux du gouvernement, un certain muselage des voix dissidentes (voir la fiche reproduite p. 95 en bas) ; des titres qui furent en leur temps interdits et le demeurent d'ailleurs toujours ${ }^{3}$, et pour lesquels la BNU se conformait à la législation; enfin (et ce ne sont pas les moins nombreux) des titres qui n'étaient frappés de nulle interdiction, mais dont les bibliothécaires de l'époque ont jugé qu'ils pouvaient être considérés comme « contraires aux bonnes mœurs » : études d'ethnographie ou d'histoire de l'art, études sur l'érotisme ou la sexologie (voir ill. p. 97 et 98). Les critères d'appréciation d'aujourd'hui feront regarder d'un œil amusé la mise à l'« enfer » de la collection Trésors inconnus, sur l'art " érotique » des civilisations anciennes, celle de l'Érotique du surréalisme de Robert Benayoun, ou encore celle de Renoir ornant la couverture du Nu dans la peinture déjà cité. Si l'absence de certains titres du catalogue, déjà évoquée dans la note 2 du présent article, doit plus à une certaine forme d'inertie des grands établissements qu'à autre chose, la présence physique, aujourd'hui encore, des titres de l'« enfer » de la BNU dans ses réserves ${ }^{4}$ relève plutôt du pittoresque et de l'insolite qu'il y a, au détour d'une recherche, à voir avoisiner Sébastien Brant, Albrecht Dürer et les pseudonymes de Thérèse ou les voluptés interdites ou de Jouir... à n'en plus finir.

\section{NOTES}

1- L'enfer de la bibliothèque : Éros au secret, exposition tenue à la BnF de décembre 2007 à mars 2008 . Un catalogue éponyme a été publié.

2- De fait, la grande majorité des fiches en question a bel et bien été retirée des catalogues usuels, ce qui fait que les ouvrages ont échappé aux diverses entreprises de rétroconversion entreprises à partir des années 90 (lesquelles n'ont pas encore concerné le catalogue de l'« enfer ») ; ils sont, du coup, absents du catalogue informatique actuel et par voie de conséquence, toujours inaccessibles au public ! La censure a la vie dure... Il est bien évident que cette anomalie devra être réparée au plus vite.

3- Voir à ce sujet Bernard Joubert, Dictionnaire des livres et journaux interdits, Paris, Éditions du Cercle de la Librairie, 2007. Pour cette raison, nous avons cru devoir nous en tenir à la stricte enquête bibliographique et ne reproduire les couvertures qu'en ce qu'elles permettent une identification claire, à l'instar du dictionnaire cité.

4- Signalons d'ailleurs que l'acquisition de documents d'intérêt régional majeur peut aussi concerner des publications clandestines ou semi-clandestines à caractère érotique. Cf. La Revue de la BNU, $\mathrm{n}^{\circ} 6,2012$, p. 104. 

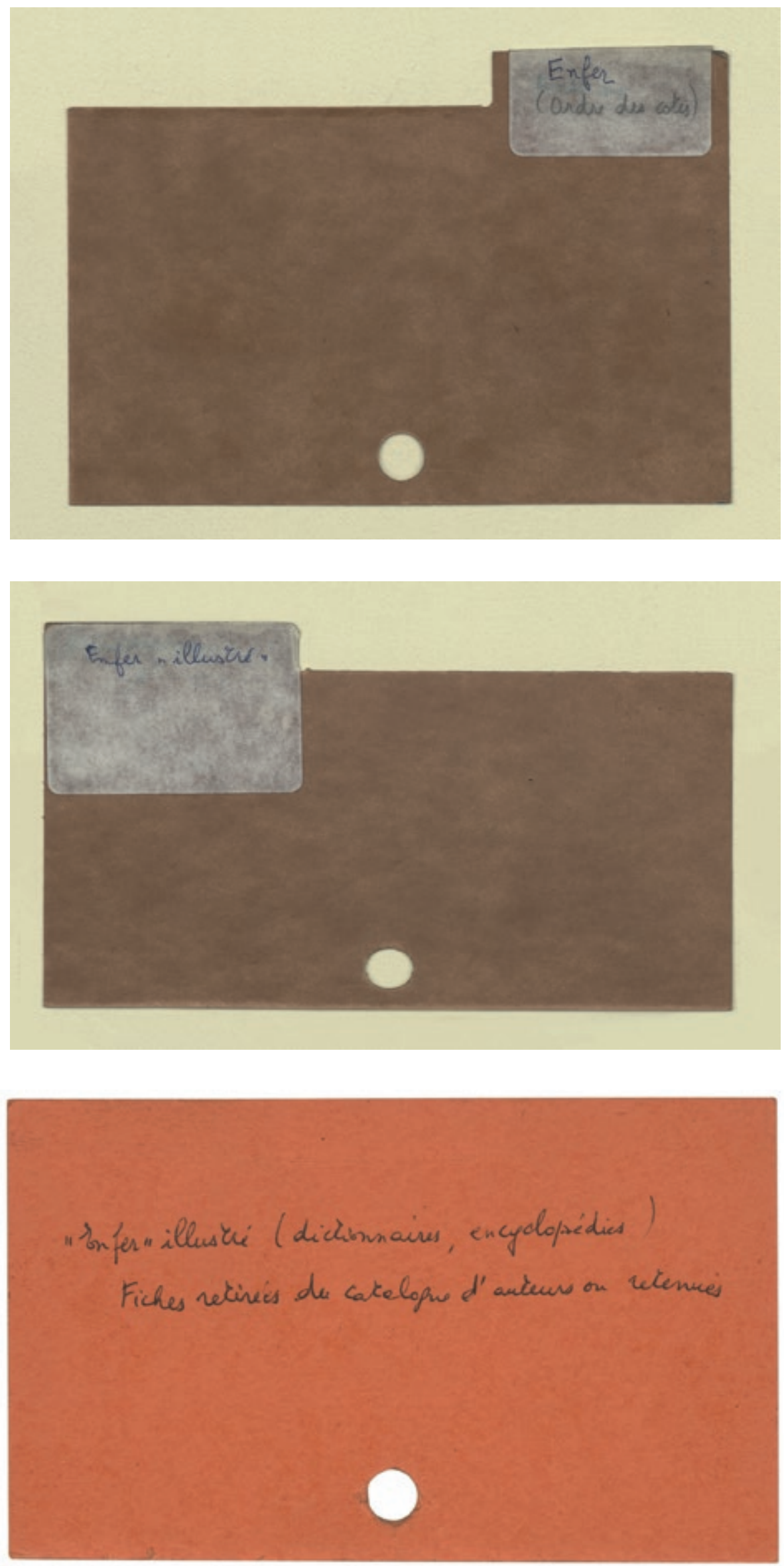
CRBBILLON, fils.- Contes dialogués... Avec une notice bio-bibliographique par Octave Uzanne.- Paris, A. Quantin, 1879.- In $-8^{\circ}$, en front. portr. de l'auteur gravé par Ad. Lalauze, vignette en taille douce, bandeaux, initiales historiées, culs de lampe, [2] pl.

(Petits conteurs du XVIIIe siècle.)

10144

$$
\text { Enfer }
$$

APOLLINAIRE (Guillaume). - Les Exploits d'un jeune Don Juan : roman / Guillaume Apollinaire; préf. de Michel Décaudin. - [Paris] : J.J. Pauvert, 1977.

- 163 p. ; $21 \mathrm{~cm}$.

ISBN 2-7202-0055-7

Décaudin (Michel). Préf.

\section{CONGO-KINSHASA. 1958-1967}

A $501852,207 / 208$ Exclu du pret. CENSURE

KAMITATU (Cléophas).- La Grande mystification du Congo-Kinshasa. Les crimes de Mobutu.- Paris, F. Maspero, 1971.- $22 \mathrm{~cm}, 304$ p., couv. illd $v(e n t b$ (Cahiers libres. 207/208.)

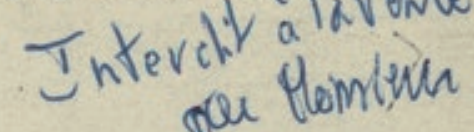

- Congo-Kinshasa. 1958-1967.- Politique. CongoKinshasa. 1958-1967. 


\title{
POINT \\ DE LENDEMAIN
}

\author{
Conte
}

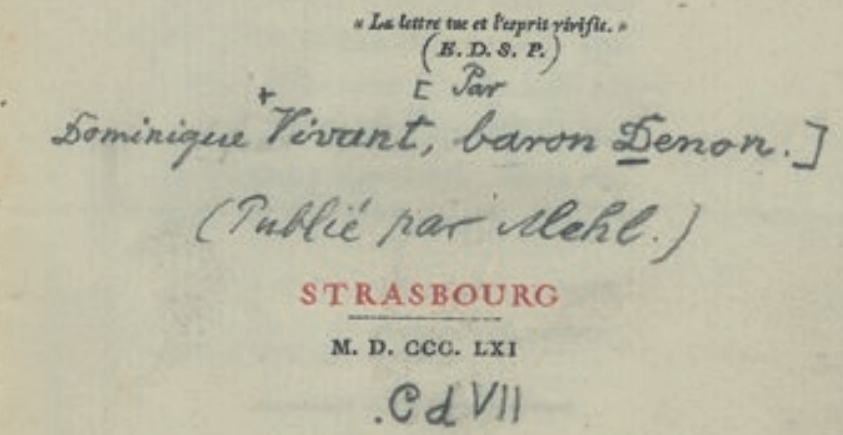

- En la

R 104929

DEXON(Dominique Vivant, baron).- Point de lendemain. Conte. [Publ. par Mehl].- Strasbourg, 1861.- In-8 ${ }^{\circ}$. (Réimpr. de 1'éd. de 1812)

21523 

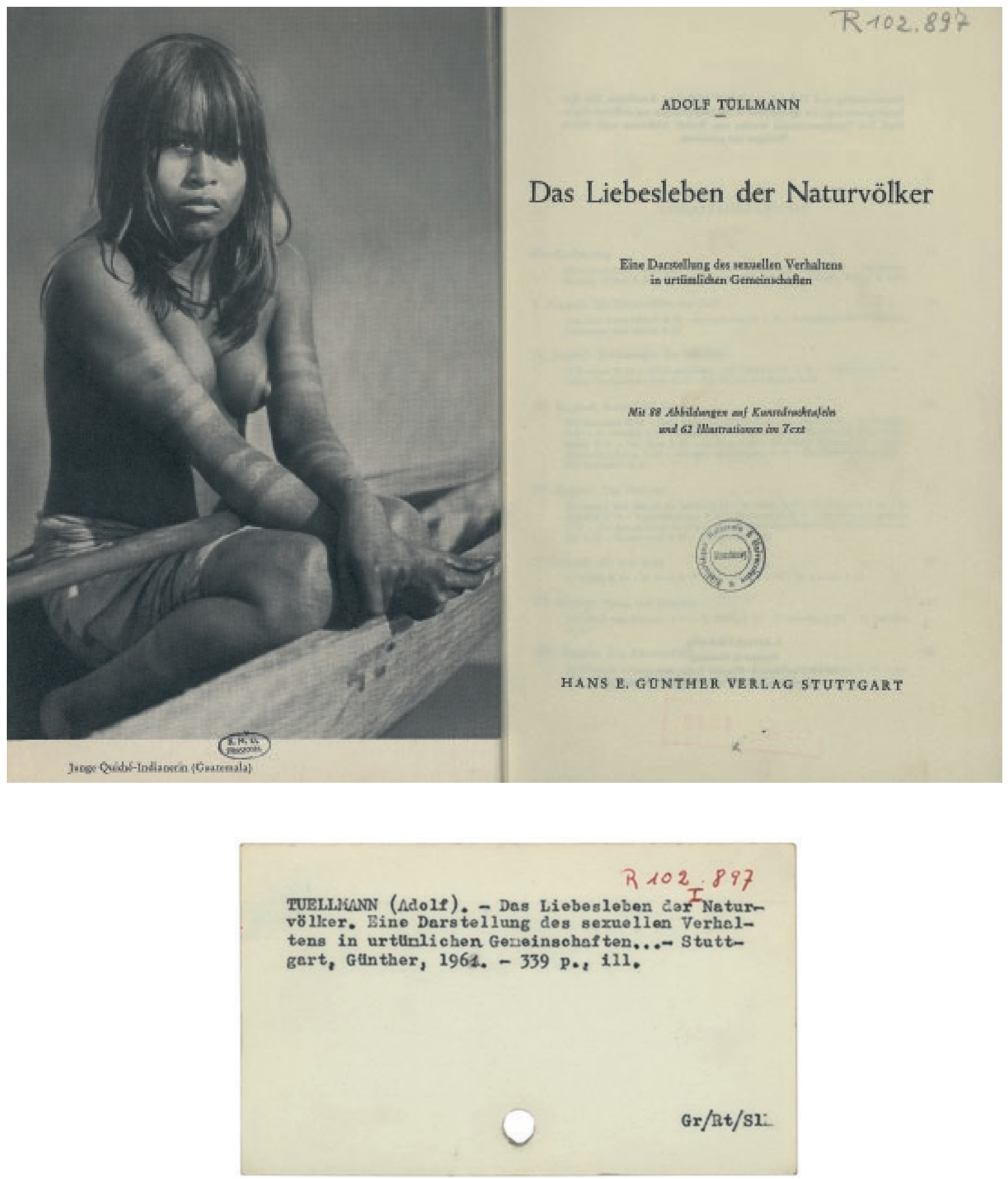


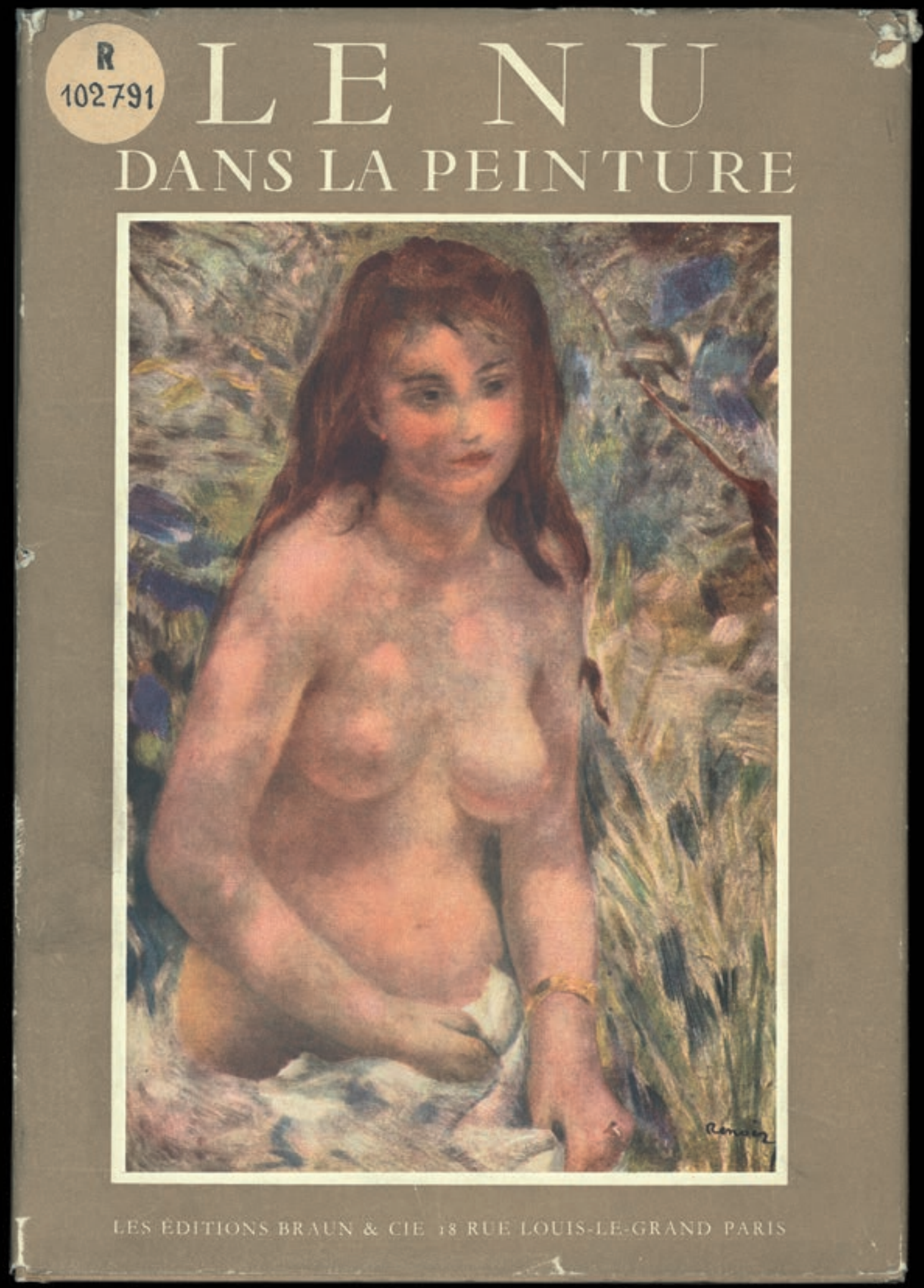




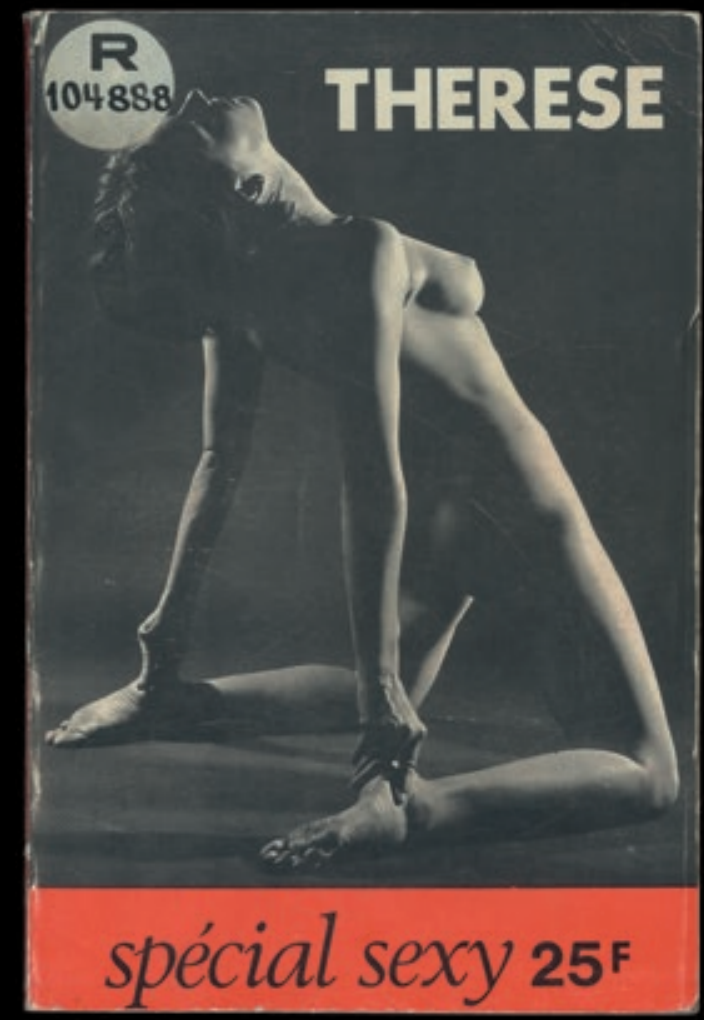

\section{$\mathbf{R} \mathrm{TS}$ \\ 104871 \\ -CUYERES DU VICE}
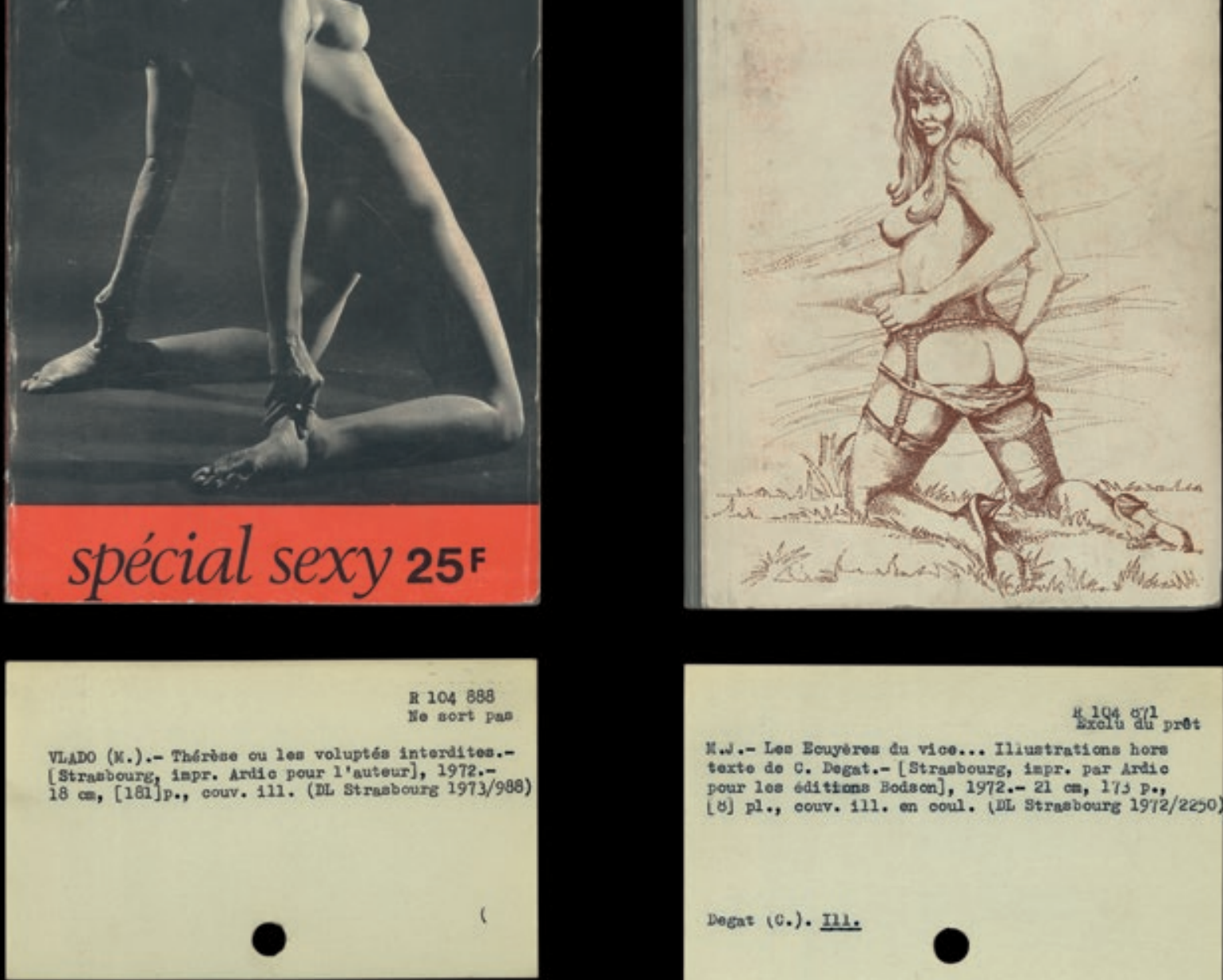

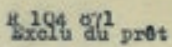

11. . .- Lee Beuybres da vice... Iliuatrations hors texte do C. Dogat.- [Strabbourg, Iepr. par Aritio

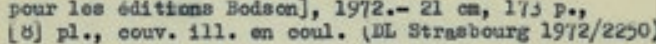

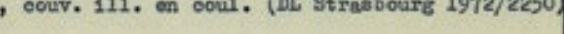

Degat (c.). 프.

R 104793

He sort pas

ULKE (Monika).- Jouir... à n'en plus finir...-

[Strasbourg, impr. Ardio pour Brotika shop],

1971.- $21 \mathrm{~cm},[137]$ p., 111., couv. 111. on

n. et bl., (DL Strasbourg 1972/250).

Sair par la enarve 